\title{
Inspection of bonding areas between two metallic plates with flash method and two temperature analyses
}

\author{
by T. Vogt Wu*, C. Pradere*, J.L. Dauvergne**, J.C Batsale*, D. Balageas* \\ *Univ. Bordeaux, I2M, UMR 5295, F-33400 Talence, France - t.vogtwu@i2m.u-bordeaux1.fr \\ ${ }^{\star *}$ Epsilon Fahrenheit, F-33400 Talence, France - jldauvergne@epsilon-alcen.com
}

\begin{abstract}
Checking the quality of bonding areas between metallic plates is crucial in many industrial fields. This paper describes a new detection method of the flaws in bonding areas between two metallic (or thermally conductive) plates by flash method. In such situations, the in-plane heat diffusion in the metallic conductive plates is blurs the defect boundary. The temperature distributions of both front and rear faces are recorded, analyzed simultaneously and combined. In this way, defects are detected more efficiently, and complementary theoretical properties (in-plane diffusivity and defect transverse resistivity) of the system are studied. An experimental example illustrates the method.
\end{abstract}

\section{Introduction}

Bonding areas are more and more common in industrial processes, for example in car design or in building engineering (double glazing or Buildings Integrated Photovoltaic Detectors for instance). These industrial systems often require quick auscultation methods in order to check the quality of the structure. Because of the in-plane thermal diffusion that blurs signals, the flaws do not appear clearly on the temperature distribution map. That is why the auscultation of such zones is difficult; moreover, the temperature contrast is small and thus hard to analyze.

The method presented in this paper gives suitable complementary theoretical properties of the material. One face of the structure is excited using a short heat pulse and both front and rear faces temperature distributions are analyzed simultaneously.

Generally the flash method (see [1]) is based on the analysis of the temperature responses of a single face of the structure. In this work, it is proposed to combine the responses of both faces in order to set out suitable new considerations. Such combined temperature responses are sometimes used in order to characterize heat transfer in buildings (see [2], [3]), but, to our knowledge, have never been used in the case of flash method.

\section{Two temperatures analysis with an homogeneous sample and extension to a 2 layered-system.}

Even if the flash method is very classically used in order to estimate the thermal diffusivity, only the temperature of one face (front or rear) is generally considered. If the simultaneous observation of the front and rear face is possible, several simple analytical expressions of the linear combination of the front and rear face temperature can then be considered. The Front face temperature at $z=0$ is expressed as $T(0, t)$ and the rear face at $z=e$ is expressed as $T(e, t)$. The analytical expressions when the sample is assumed to be adiabatic after the pulse and initially at zero temperature can be obtained versus the thickness: $e$ of the sample, the thermal diffusivity: a the areal capacity $\rho c e$ and the energy $Q$ in $\mathrm{Jm}^{-2}$, such as:

$$
\left\{\begin{array}{l}
T_{1}(t)=T(0, t)=\frac{Q}{\rho c e}\left(1+2 \sum_{n=1}^{\infty}(1)^{n} \exp \left(-n^{2} \pi^{2} a t / e^{2}\right)\right. \\
T_{2}(t)=T(e, t)=\frac{Q}{\rho c e}\left(1+2 \sum_{n=1}^{\infty}(-1)^{n} \exp \left(-n^{2} \pi^{2} a t / e^{2}\right)\right.
\end{array}\right.
$$

Then, the sum (Eq. 3.) and the difference (Eq. 4.) of front and rear faces analytical solutions can be obtained by the elimination of the pair or non-pair terms of the series, such as:

$$
\left\{\begin{array}{c}
T_{1}(t)+T_{1}(t)=T(0, t)+T(e, t)=\frac{2 Q}{\rho c e}\left(1+2 \sum_{k=1}^{\infty}(1)^{k} \exp \left(-4 k^{2} \pi^{2} a t / e^{2}\right)\right. \\
T_{1}(t)-T_{1}(t)=T(0, t)-T(e, t) \approx \frac{4 Q}{\rho c e}\left(\exp \left(-\pi^{2} a t / e^{2}\right)+\exp \left(-4 \pi^{2} a t / e^{2}\right)+\ldots\right)
\end{array}\right.
$$


Their asymptotic development for long time behavior $\left(t>\mathrm{e}^{2} /\left(4 \pi^{\star} \mathrm{a}\right)\right)$ leads to the following approximations:

$$
\left\{\begin{array}{c}
T_{1}(t)+T_{2}(t)=T(0, t)+T(e, t) \cong \frac{2 Q}{\rho c e} \\
T_{1}(t)-T_{2}(t)=T(0, t)-T(e, t) \cong \frac{4 Q}{\rho c e} \exp \left(-\frac{\pi^{2} a t}{e^{2}}\right)
\end{array}\right.
$$

The sum $T_{1}(t)+T_{2}(t)$ is constant and the difference $T_{1}(t)-T_{2}(t)$ is exponential.

The long time behavior of the front and rear faces signals sum (5.) represents the front face response after a flash applied to a e/2-thick sample, with an adiabatic rear face. The long time behavior of the difference (6.) represents a front face response after a flash applied to a e/2-thick sample, with an isothermal rear face (imposed zero temperature at the rear face). These analytical results are summarized in the following figure (Fig. 1.):

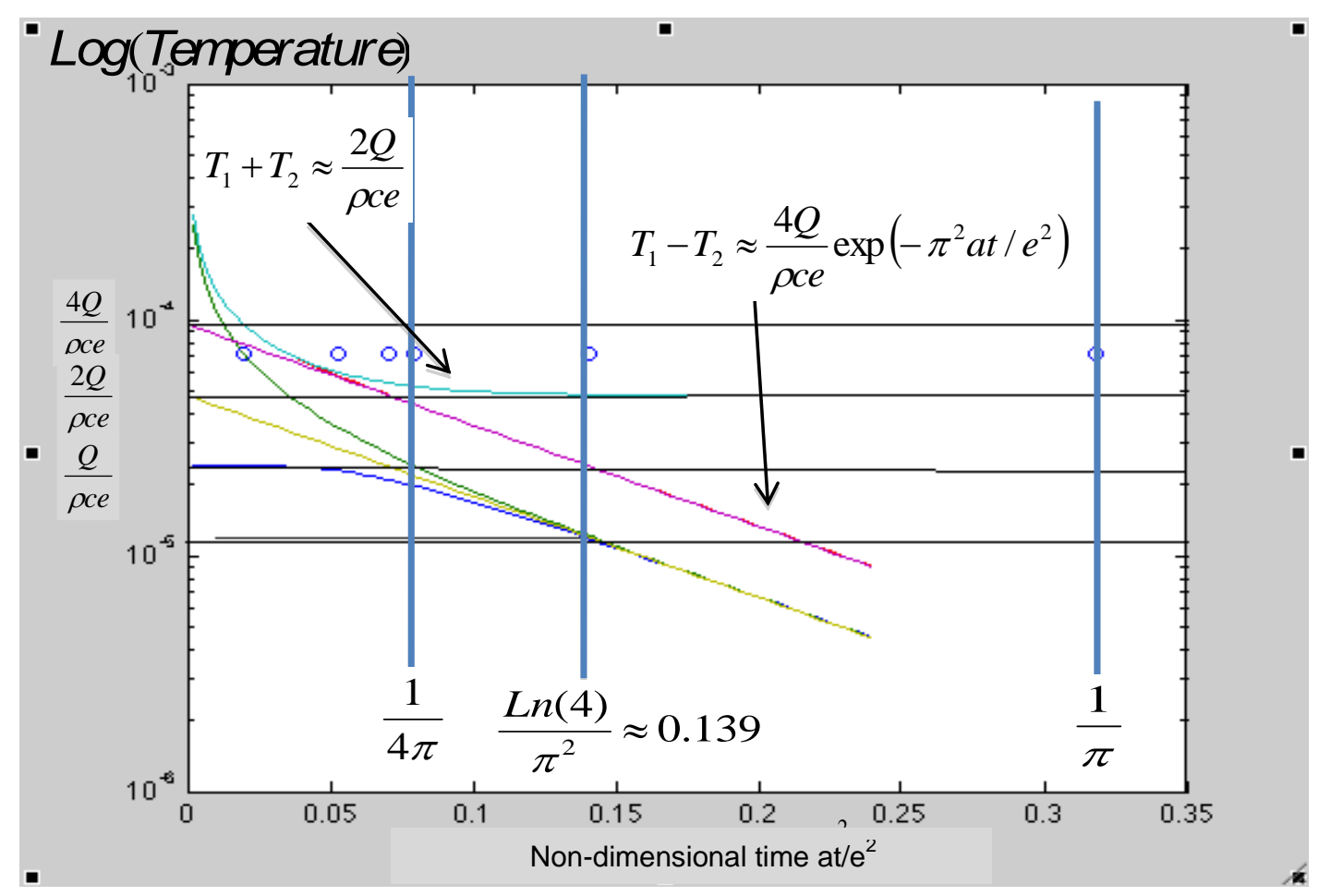

Fig. 1. Analytical responses of the temperature differences $T_{1}(t)-T_{2}(t)$ (purple) and temperature sum $T_{1}(t)+T_{2}(t)$ (light blue) in a Log (Temperature) versus a non dimensional time scale presentation.

Translated front and rear face thermograms: $T_{1}(t)-\frac{Q}{\rho c e}$ (green), $\frac{Q}{\rho c e}-T_{2}(t)$ (dark blue), $T_{1}(t)-T_{2}(t)-\frac{Q}{\rho c e}$ (yellow).

It is here important to note that the characteristic time of $T_{1}(t)-T_{2}(t)$ and $T_{1}(t)+T_{2}(t)$ are about $1 / 4 \pi$ earlier than the classical characteristic time of the front face thermogram: $T_{1}(t)$ which is $1 / \pi$ and the rear face thermogram : $T_{2}(t)$ which is about $\operatorname{Ln}(4) / \pi^{2} \cong 0.139$. Such linear combinations of the front and rear face thermograms are then giving a suitable approximations at earlier time $(>4 / \pi$ instead of $1 / \pi)$.

Thanks to the exponential behavior of $T_{1}(t)-T_{2}(t)$ and if $t>\operatorname{Ln}(2) e^{2} / \pi^{2} / a$, a suitable approximation of the front and rear face thermograms can be: 


$$
\left\{\begin{array}{l}
T_{1}(t)=\left(T_{1}(t)+T_{2}(t)+T_{1}(t)-T_{2}(t)\right) / 2 \equiv \frac{Q}{\rho c e}+\frac{2 Q}{\rho c e} \exp \left(-\pi^{2} a t / e^{2}-\operatorname{Ln}(2)\right) \\
T_{2}(t)=\left(T_{1}(t)+T_{2}(t)-\left(T_{1}(t)-T_{2}(t)\right)\right) / 2 \equiv \frac{Q}{\rho c e}-\frac{2 Q}{\rho c e} \exp \left(-\pi^{2} a t / e^{2}-\operatorname{Ln}(2)\right)
\end{array}\right.
$$

It is then possible to consider such solutions as the solution of capacity-resistance-capacity analogical circuit (see figure 2 or Maillet et al, 2000).

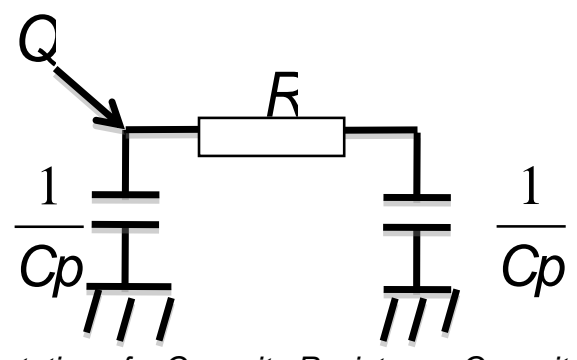

Fig. 2. Analogical representation of a Capacity-Resistance-Capacity circuit.

Such a modeling is very similar to the bonding problem between metallic plates. The plates are considered as capacitive and isothermal; the thermal resistance is related to the bonding of the thin air layer that is between the two plates. The front and rear face plates are thus verifying the following first order ordinary differential system :

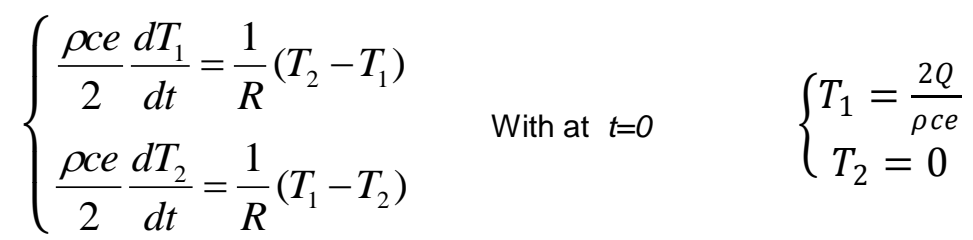

Where the capacity and resistance definition are: $C=\rho c e / 2$ and $R=4 e / \pi^{2} \lambda$.

Such approximations can be adapted to flash experiments in the case of non-uniform bonding. The in-plane diffusion must then be taken into account and the temperature fields can be considered as depending on $x, y$ coordinates or $I, j$ pixels of a temperature field analyzed by infrared camera. Each pixel of the temperature response map of the front and the rear face (figure 1) can then be written respectively as:

$$
\begin{gathered}
\rho c e \frac{\partial T_{1}}{\partial t}=\lambda e\left(\frac{\partial^{2} T_{1}}{\partial x^{2}}+\frac{\partial^{2} T_{1}}{\partial y^{2}}\right)+\frac{1}{R(x, y)}\left(T_{2}-T_{1}\right)-h T_{1} \\
\rho c e \frac{\partial T_{2}}{\partial t}=\lambda e\left(\frac{\partial^{2} T_{2}}{\partial x^{2}}+\frac{\partial^{2} T_{2}}{\partial y^{2}}\right)+\frac{1}{R(x, y)}\left(T_{1}-T_{2}\right)-h T_{2}
\end{gathered}
$$

It is then suitable to consider the sum and the difference of the temperature responses $\left(T_{1}+T_{2}\right)$ and $\left(T_{1}-T_{2}\right)$. Indeed, the influence of the contact resistance $R(x, y)$ is eliminated when summing the temperatures of face 1 and 2 (Eq. (2-a)); therefore only the global heat losses $h / \lambda e$ and the in-plane thermal diffusivity $\lambda / \rho c$ are to be taken into account and estimated.

When considering the temperature difference, Eq. (11-b), the influence of the contact resistance is correlated with the in-plane thermal diffusivity.

$$
\begin{gathered}
\rho c e \frac{\partial\left(T_{1}+T_{2}\right)}{\partial t}=\lambda e\left(\frac{\partial^{2}\left(T_{1}+T_{2}\right)}{\partial x^{2}}+\frac{\partial^{2}\left(T_{1}+T_{2}\right)}{\partial y^{2}}\right)-h\left(T_{1}+T_{2}\right) \\
\rho c e \frac{\partial\left(T_{1}-T_{2}\right)}{\partial t}=\lambda e\left(\frac{\partial^{2}\left(T_{1}-T_{2}\right)}{\partial x^{2}}+\frac{\partial^{2}\left(T_{1}-T_{2}\right)}{\partial y^{2}}\right)+\frac{1}{R(x, y)}\left(T_{1}-T_{2}\right)-h\left(T_{1}-T_{2}\right)
\end{gathered}
$$


In order to study several estimation processes it is here proposed to examine the properties of the images obtained from a simple experiment with a 2-layered metallic sample.

\section{Illustration with a 2-layered sample experiment.}

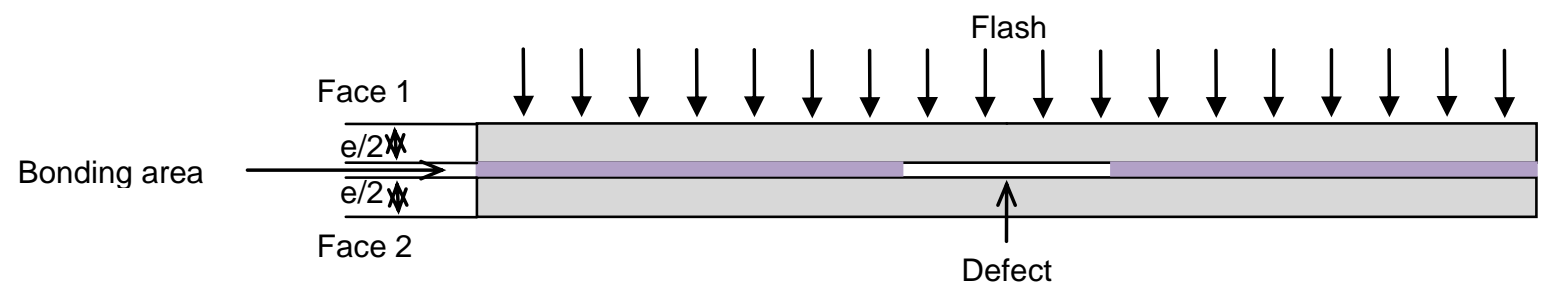

Fig 3. Experimental principle

Two aluminium plates (of thickness $e / 2=0.6 \mathrm{~mm}, I=90 \mathrm{~mm}, L=90 \mathrm{~mm}$ ) are uniformly sticked excepted on a rectangular area of $b=12.2 \mathrm{~mm}$ width and $L=90 \mathrm{~mm}$ length. Such area is an air layer assumed to be insulating and of thickness about $0.1 \mathrm{~mm}$.

A heat pulse (flash) is imposed on the front face of the sample and the temperature response is measured respectively on the front and rear face of the sample, versus time. The time step value for each frame recording is $0.01 \mathrm{~s}$. The ideal experimental condition would be to measure simultaneously the front and rear face temperature distributions. For practical reasons, the data are obtained with two experiments where the camera is alternatively measuring the two faces. The thermograms are then normalized versus the long time behavior, where the field is quite uniform and steady.
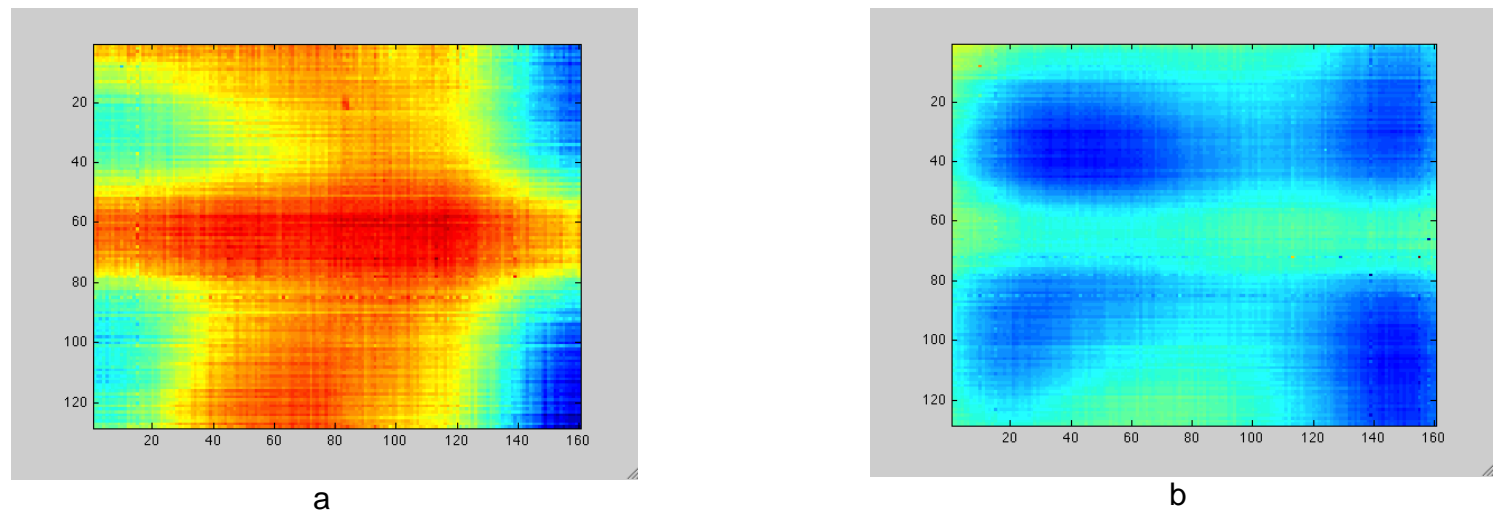

Fig 4. Instantaneous temperature image at time $t=174^{*} 0.01 \mathrm{~s}$. $a$ - front face: $b$ - rear face: T2

It can be observed on figure 4 that the front and rear face observations are affected by the in-plane thermal diffusion, even if the defect can be detected (horizontal layer).

One illustration of the $\left(T_{1}+T_{2}\right)$ and $\left(T_{1}-T_{2}\right)$ observations are given on figure 5 . It can be noticed that the $\left(T_{1}+T_{2}\right)$ observation is quite uniform (conformal to the theory). The $\left(T_{1}-T_{2}\right)$ is less noisy and slightly more contrasted than the respective $T_{1}$ and $T_{2}$.
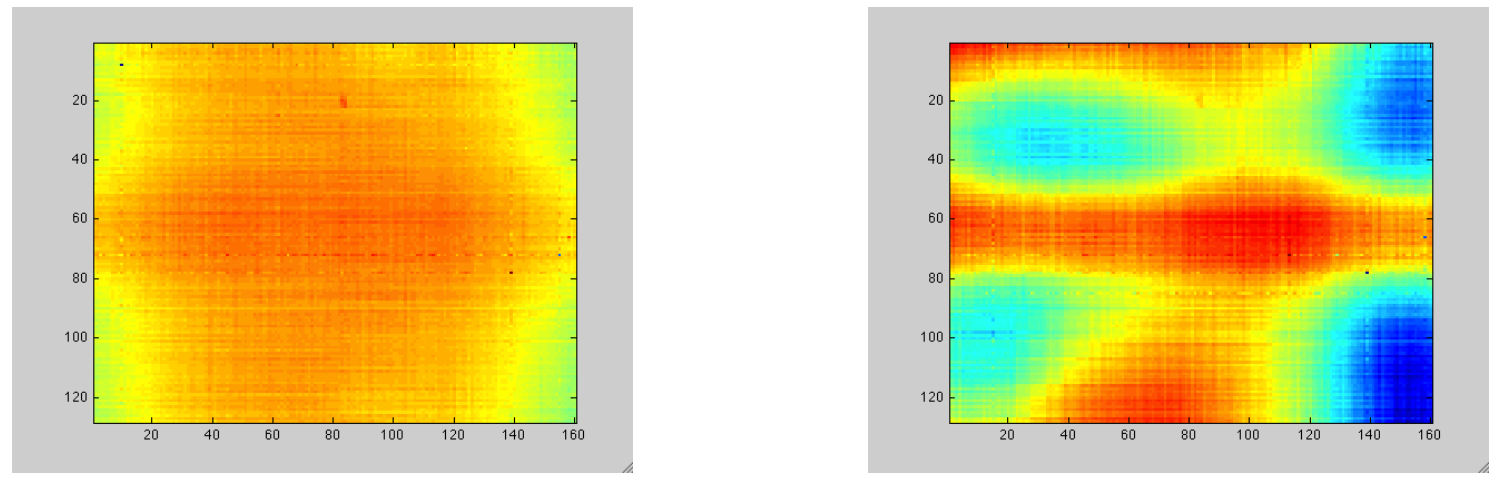

Fig 5. Instantaneous combined temperature images at 174 time steps. The time step value is $0.01 \mathrm{~s}$. Left from the front face $T 1+T 2$ and right from the rear face. 
In our experimental study, the system is nearly one-dimensional, so we can also consider here only the $x$ direction while averaging along the $y$ direction. One example of experimental result obtained with two plates of aluminum partially bonded is then given on figure 6 . It can be observed that the defect does not appear on the normalized $\left(T_{1}+T_{2}\right)$ profile, however the normalized $\left(T_{1}-T_{2}\right)$ profiles are clearly showing the blurred defect position.

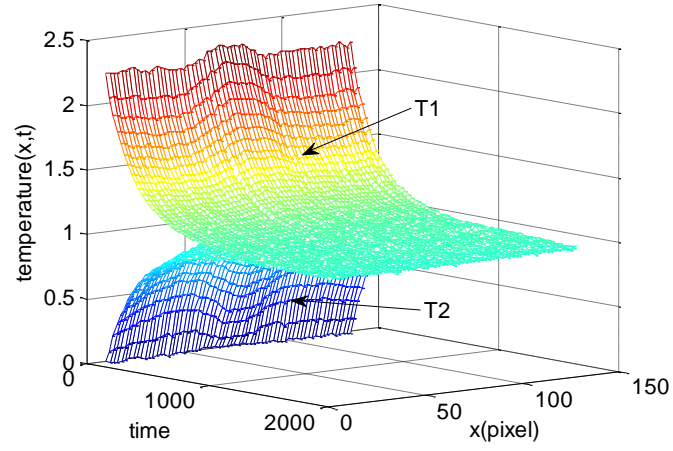

Figure. 6.a. Front and rear face temperature versus time and $x$

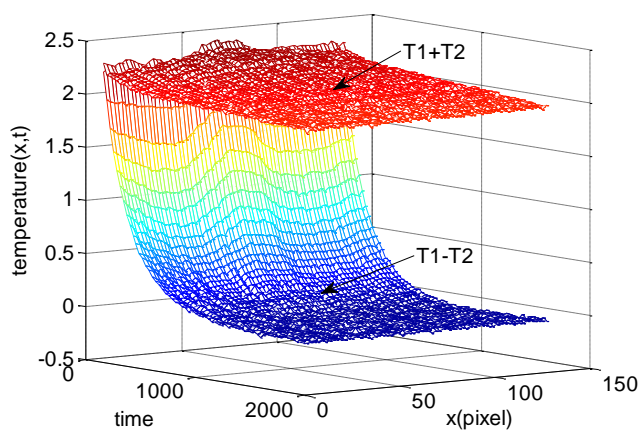

Figure.6-b. Temperature sum and difference of the front and rear face evolution versus time and $x$

From the previous analytical considerations, the plotting of $T_{1}-T_{2}$ on a logarithmic scale allow to verify the globally exponential behavior (see figure 7). The curves corresponding to each $\mathrm{x}$ value are near from straight lines.

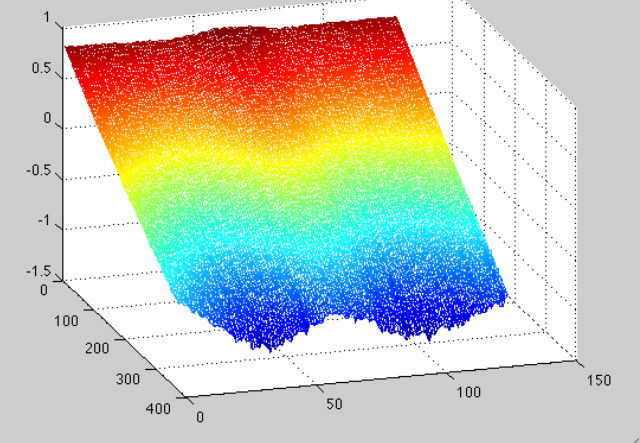

Fig 7: Plotting of $\log \left(T_{1}-T_{2}\right)$ versus time (400 time steps represented) (x pixels from 1 to 126)

\section{4._Estimation strategy}

A lot of strategies can be considered in order to estimate the thermal resistance distribution $R(x, y)$.

One strategy can be to consider a purely transverse heat transfer model without in plane diffusion. Each pixels are then considered as non correlated with the neighborhood. It would here consist to measure the time slope of each $x-$ curve related to $\log (T)$ on the figure 7 . Unfortunately the blurring effects due to in-plane diffusion are affecting the estimation. It can be seen on figure 8 that the edge effect are affecting the estimation results.

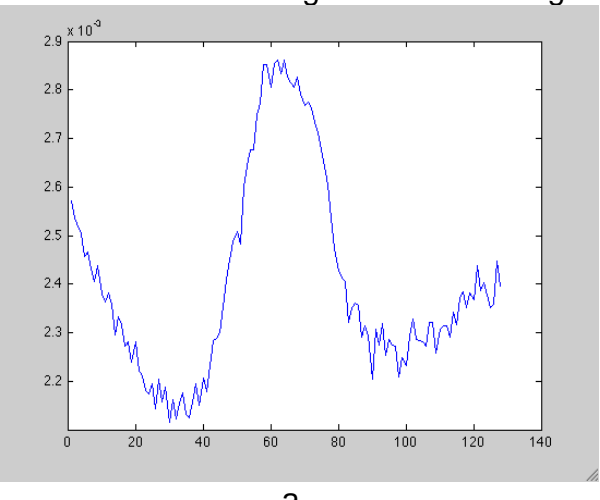

a

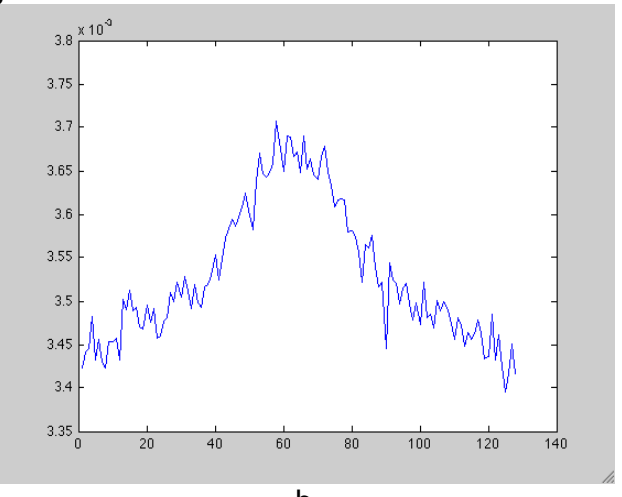

b

Fig 8: Estimation of the time slope of $\log \left(T_{1}-T_{2}\right)$ and $\log \left(T_{1}+T_{2}\right)$ versus $x$ in the 1D case. 
Another strategy is to consider only the areas where the time behavior is related to a pure capacitive discharge or where: $\frac{d\left(T_{1}-T_{2}\right)}{d t}$ is proportional to $\left(T_{1}-T_{2}\right)$. such as:

It consists in considering the correlation coefficient between the time derivative of the signal and the signal itself

$$
\rho=\frac{\left.\sum_{k=1}^{N t k} \frac{d\left(T_{1}-T_{2}\right)}{d t}\right|_{t k}\left(T_{1}-T_{2}\right)(t k)}{\left(\sum_{k=1}^{N t k}\left(\left.\frac{d\left(T_{1}-T_{2}\right)}{d t}\right|_{t k}\right)^{2}\right)^{1 / 2}\left(\sum_{k=1}^{N t k}\left(\left(T_{1}-T_{2}\right)(t k)\right)^{2}\right)^{1 / 2}}
$$

Here the temperature is decreasing versus time. Such a correlation coefficient will be negative. If the coefficient is close to -1 , it means that the model is purely capacitive, with poor in plane diffusive effects. If the correlation is near 0 , the model is then biased. In our case, when the correlation is near 0 or lower than the neighborhood, such a parameter can be a measure of the amplitude of the in plane diffusivity effects.

The result of such a calculation on the previous data is illustrated by the mapping of correlation given on figure 9 .

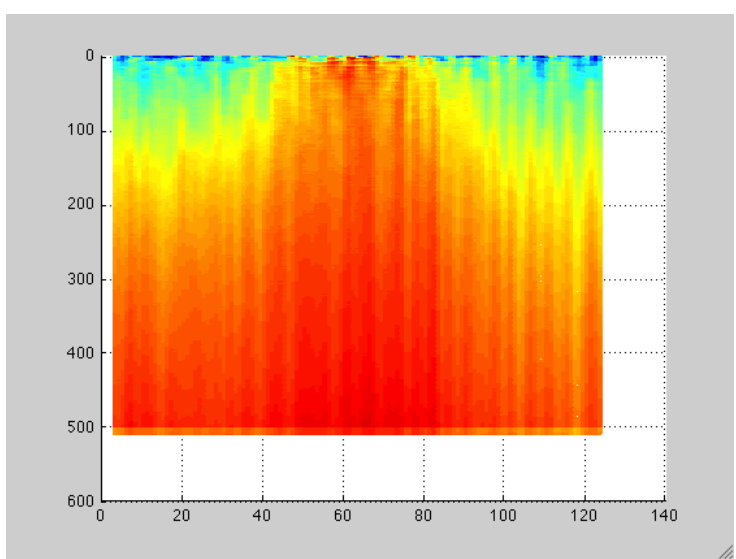

Fig 9: Correlation coefficient from 5 to 500 time steps. The red points are related to bad correlated signal and the blue ones are related to well correlated signal. (abscissa: $x$ coordinate, ordinate: correlation coefficient at 5 to 500 time steps) considered.

It appears from figure 9, that only at short time and only out of the defect the purely capacitive behavior is to be

Such a method of mapping has been applied for each pixels of the temperature field in the 2D configuration and shown in the case of $\left(T_{1}-T_{2}\right)$ signal (see figure 10). Of course, the signal/noise ratio of such an image is less favorable than on the figure 4 and 5 , and the magnitude of the values is not related to a physical grandeur. Nevertheless, such a mapping is related to a model validity and can be useful in order to test several assumptions of the models.

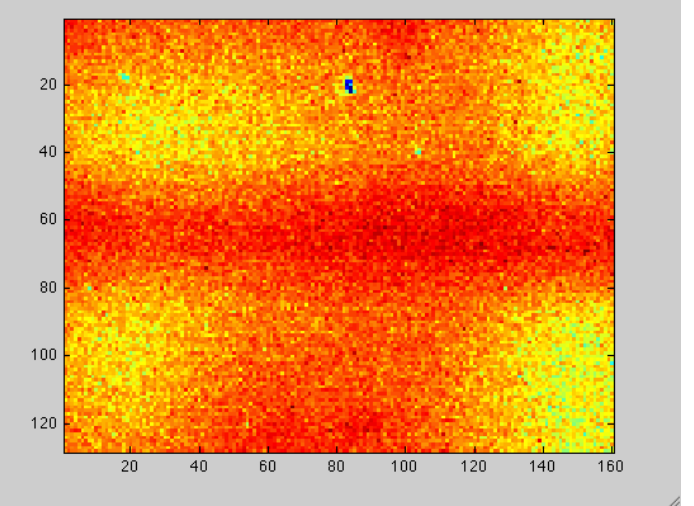

Fig 10: Correlation coefficient at 100 time steps (expression 12) for each pixel of the temperature field. The red points are related to bad correlated signal and the yellow are related to better correlated signal. 
Similarly as the figure 10 the $1 \mathrm{D}$ case is illustrated in figure 11. It appears that the area related to the defect position is less correlated than the area outside the defect. Of course such values are greater than -1 and lower than 0.

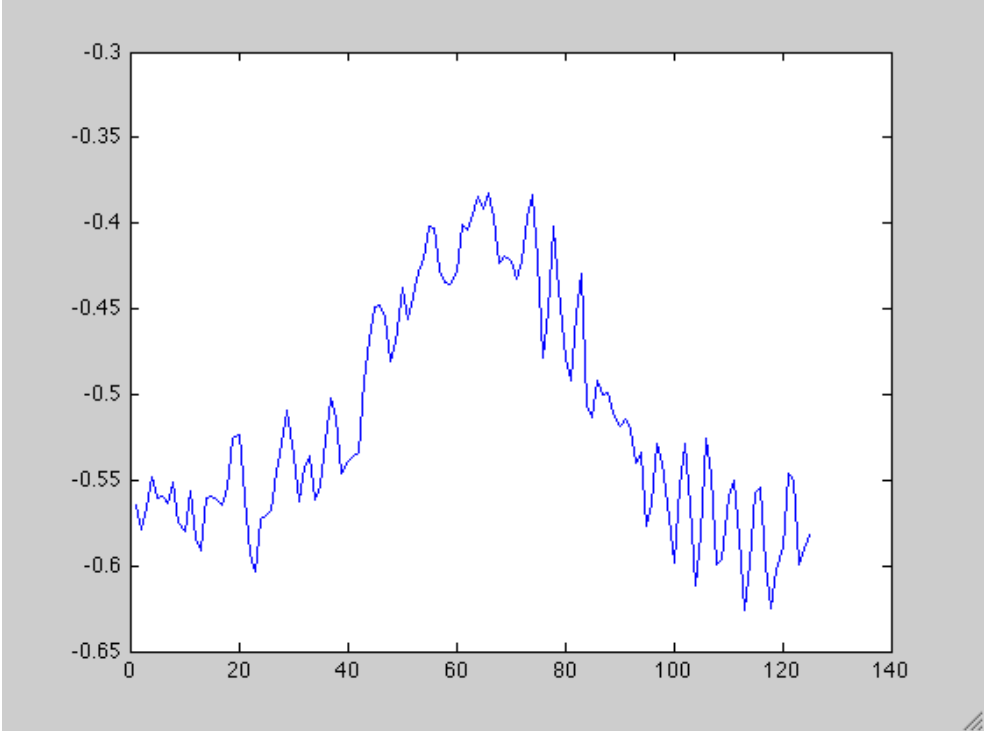

Fig 11: Correlation coefficient at 100 time steps (expression 12) for each pixel of the 1D temperature field.

\section{Conclusion}

The two temperatures analysis (front and rear face in flash experiments) allows the use of simple approximations of heat transfer such as capacity-resistance-capacity models.

In the problem of detection of bonding between two metallic plates and with no in-plane diffusion the analytical approximations suitable with purely exponential time behaviors.

In order to avoid the blurring effects due to in-plane diffusion it has been proposed to analyze the correlation between the time derivative of the signal and the signal itself (front-rear face temperature difference). It appears that the temperature field on the defect is less correlated than the temperature field related to the well bonded area. Such a method can be a way to improve the contrast and detect the defect limit without considering the in plane heat diffusion.

\section{REFERENCES}

[1] W.J. Parker, W. Jenkins, J. Abott, "Flash method of determining thermal diffusivity, heat capacity and thermal conductivity". J ppl Phys 32(9): pp. 1679-84, 1961

[2] E. Antczak, D. Defer, M. Elaoami, A. Chauchois, B. Duthoit "Monitoring and thermal characterization of cement matrix materials using non destructive testing". Non Destructive Testing and Evaluation International, Vol. 40, Issue 6, pp. 428-438, 2007

[3] T. Wu, E. Antczak, D. Defer, T. Chartier "Thermal characteristics in situ monitoring of detached house wall constituted by raw clay". European Journal of Environmental and Civil Engineering, Vol.14-No.5/2010, pp.653-667, 2010

[4] D. Maillet, S. Andre, JC Batsale, A. Degiobvani, C. Moyne, Themal quadrupole, solving the heat equation trough integral transforms, Wiley, 2000. 